\title{
INFECTIVE ENDOCARDITIS IN CHILDREN - CLINICAL AND OUTCOME EVOLUTION
}

\author{
Marinov L., P. Shivachev \\ Department of Pediatrics \\ Medical University "Prof. Dr Paraskev Stoyanov"-Varna, Bulgaria
}

\section{SUMMARY}

For a 15-year period (1992 - 2007) in the clinics of the department of pediatrics were hospitalized 10 children with endocarditis from 1 to 13 years of age. One of the children was hospitalized twice during a period of two years.

All children were with preceding congenital defects: ventricular septic defects in 5 cases; valvular aortic stenosis in 1 case; transposition of the great vessels - 1 case; hyperthrofic cardiomyopathy - 1 case; ventricular-atrial anastomosis in two child with internal hydrocephaly. Three of the cases were patients with prosthetic endocarditis.

In blood cultures were established Streptococcus viridans in 3 cases; Streptococcus 6 - haemoliticus in 2 case; Staphylococcus epidermidis in 2 cases and Staphilococcus aureus in 1 case. In $84.44 \%$ of the cases were found positive blood cultures before starting the antibacterial therapy. In patients who had received antibiotics before $-18.17 \%$ were with positive cultures.

With echocardiography was detected vegetation in 8 (73.27\%) cases.

In the course of the disease were found the following complications: cardiac deficiency - in 7 children, pleuropericardial effusions - in 4 cases, septic embolism in 3 patients and purulent mediastinitis - 1 case. Three of our IE cases have had a lethal outcome $(27,3 \%)$.

Key words: Infective endocarditis, children, clinical evolution, complications

The impact of endocarditis in childhood accompanies many cardio-vascular disorders. Infective endocarditis (IE) is the most frequently occurring form of endocardium inflammation. Prevailing among the agents are Str. Viridans, Str. Haemoliticus, Staph. Aureus, Staph. Epidermidis, Enterococcus, etc. Expression is different, mainly acute. IE is normally a complication disorder based on preceding congenital cardiopathy or operated heart $(3,5)$. Bacteria deposit and multiply on heart's endocardium or vessels' endothelium. Endocarditis could become the source of infection and bacteriaemia.

Approximately $10 \%$ of all IE cases are with children below the age of $10(4,6)$.

The clinical evolution and outcome depend to a great extent on the individual child organism, the ability of a child's immune system to respond to the increased demands, the age, and the general health of a child.

Infective Endocarditis remains a serious issue in early age. A few factors come to support this worrying statement: the disorder occurrence over the past few years has become more frequent, in relation to the larger number of children with heart operations; protracted and expensive treatment; complications as a result from the disorder, sometimes including a re-operation, and a still high rate lethality.

\section{AIM} in children.

Retrospective analysis of IE evolution and outcome

\section{MATERIALAND METHODS}

For a 15-year period $(1992-2007)$ in the clinics of the Pediatrics department we have observed 11 IE cases in 10 children at the age from 1 to 13. Six of the children are female, and four - male. One of the children has been diagnosed twice with prosthetic endocarditis caused by various infection agents.

The children have been clinically, paraclinically and instrumentally tested, the decisive being septic condition indices, as well as the analyses of blood cultures and echocardiography.

\section{RESULTS AND DISCUSSION}

All children with IE have a preceding cardiac lesion. In 7 of the cases we have had congenital cardiac malformations - 5 children with ventricular septal defects (VSD); 1 with valvular aortic stenosis (AoVSt) and 1 with transposition of the great arteries (TGA), operated under Rastelli. One child had a family obstructive hyperthrophic cardiomyopathy (HCMP); and two a ventricular-atrial anastomosis (VAA) based on internal hydrocephaly. In three of the cases we had prosthetic IE.

In all of the cases there was a septic condition with continual febrility, weakness, lack of appetite, difficulty in breathing, vomiting, abdominal pains, skin rash, arthralgia, hepato- splenomegaly, and paraclinical indices for high inflammatory activity.

The haemocultural analysis proved the following bacterial agents: Str. viridans in 3 children, Str. $\alpha-$ 
haemoliticus in two children, Staph. aureus in 1, and Staph. epidermidis - in 2 children. It is important to point out that we have had in $84.44 \%$ of the cases positive haemocultures inoculation prior to the start of anti-bacterial treatment $(22$ out of 26 tests) and only in $18.17 \%$ (4 out of 22 inoculations) post anti-bacterial treatment.

Vegetations was detected by echocardiography in 8 of the IE cases (73.27\%), located around the cardiac lesion (Fig. 1. and Fig. 2.).

The main complications observed with us are as follows: heart failure in 7 children; pericardial and pleural effusions - in 4 cases; lung or system embolism - in 3 and purulent mediastinitis - 1 case. In some of the children we have encountered complications. For that reason the whole amount of the complications sum more than $100 \%$. children

Operative treatment was necessary in 3 of the

Three of our IE cases have had a lethal outcome $(27,3 \%)$. The average pre-hospitalization period in cured

Fig. 1. Vegetations located around the cardiac lesion

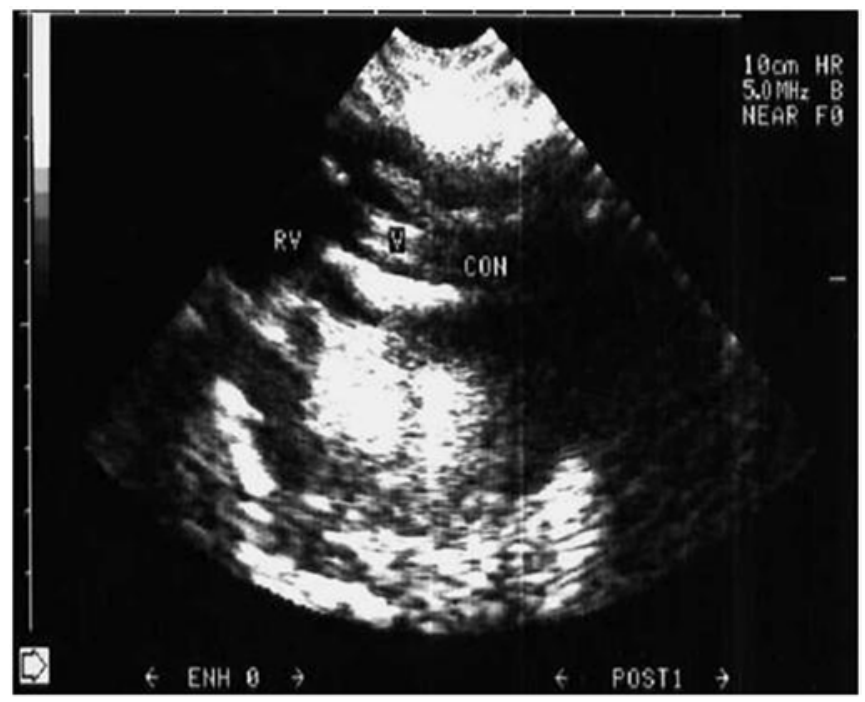

Table 1. Preceding cardiac lesions disorder

\begin{tabular}{|l|c|}
\hline Preceding lesions & Number \\
\hline VSD & 5 \\
\hline AoVSt & 1 \\
\hline TGA & 1 \\
\hline HCMP & 1 \\
\hline VAA & 2 \\
\hline
\end{tabular}

children was 23, against 35 days in the deceased children.

Results show that in our survey children age up to 13 years. As already noted above, the highest frequency was observed in children up to 10 years of age. There has been no significant difference observed between the two sexes. As other authors note the most common agents isolated out of haemocultural analyses are Str. Viridans and Staph. Aureus $(1,7,8,9,10)$. In over $80 \%$ of the tests positive haemocultures were found prior to the start of anti-bacterial treatment, which is understandable. Of a much lower rate were the positive haemocultures in tests done post anti-bacterial treatment.

With all children IE has emerged on the basis of a preceding cardiac lesion, and in our case a larger share is attributed to ventricular septum defect (VSD) - in 5 cases and on one occasion again after operation based on non-cardiac disorder. The allocation of children with preceding cardiopathy is shown on Table 1. Haemocultural analysis results are shown in Table 2.

Fig. 2 . Vegetations located around the cardiac lesion

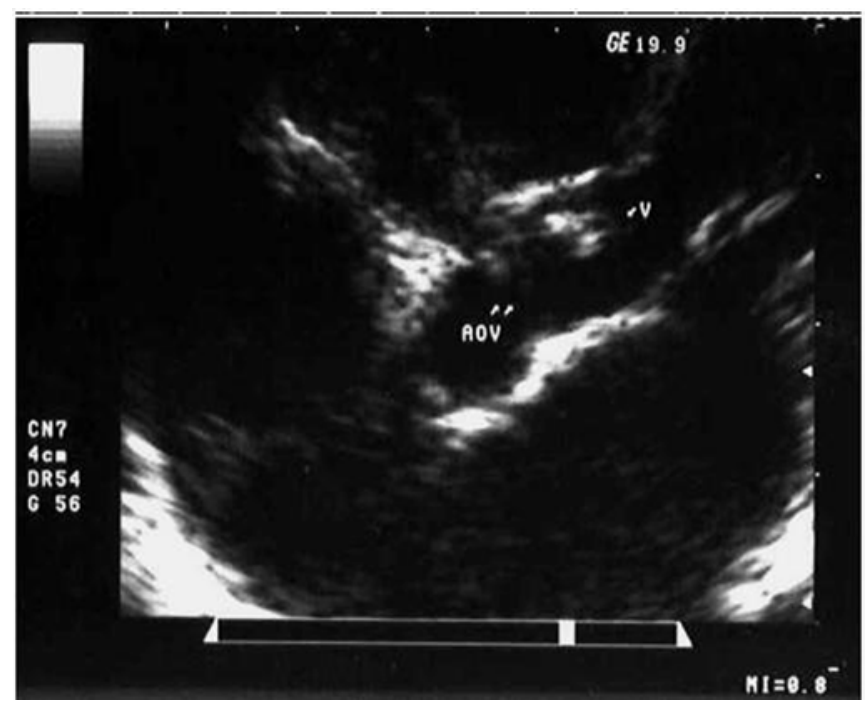

Table 2. Results from the haemocultural analysis

\begin{tabular}{|l|c|}
\hline Bacterial agent & Number \\
\hline Str. viridans & 3 \\
\hline Str. $\alpha$ - haemoliticus & 2 \\
\hline Staph. epidermidis & 2 \\
\hline Staph. aureus & 1 \\
\hline
\end{tabular}


Table 3. Complications developing in the course of disorder.

\begin{tabular}{|l|c|}
\hline Complications & Number \\
\hline Heart failure & 7 \\
\hline Pericardial and pleural effusion & 4 \\
\hline Thromboembolisme & 3 \\
\hline Mediastinitis & 1 \\
\hline
\end{tabular}

Despite the timely implementation of anti-bacterial and symptomatic treatment, complications in the course of the disorder did occur. The complications occurring in the course of disorder are shown in tab. 3.

The leading complication in 7 of the cases was cardiac deficiency. This is explanatory in view of the preceding congenital cardiopathy and the general prejudicial condition of the children. The remaining complications - the presence of pericardial and pleural effusions we managed conservatively. The most dramatic and involving hard effort was our battle to contain the cases of embolism and mediastinitis development in one of the cases, where an operation "on the hot" became necessary. Other authors also inform of dramatic development and fatal outcome of the same and other complications - stroke in seven children $(2,9)$.

Under the circumstances, despite the appropriate symptomatic and surgical treatment, death rate in children with IE remains high. In our survey we have registered lethality of $27.3 \%$, which corresponds to data from other authors (6). The most common complication, resulting directly into a lethal outcome is the development of a heavy cardiac deficiency in $70 \%$ of the cases. The most dramatic and hard was the evolution of the disorder in the presence of system and lung embolism, quoted by other authors (2).

In conclusion it should be said that IE remains still as a problem for contemporary medicine and in particular pediatric cardiology and cardio surgery.

The results from our retrospective study allow us to draw the following conclusions: $27.3 \%$

1. IE is a serious disease with lethality in our cases of

2. There is a presence of a preceding cardiac lesion.

3. The most common complication is cardiac deficiency (70\%), and the hardest one - system and lung embolism.

\section{REFERENCE:}

1. Barreira JL, Baptista MJ, Moreira J., et al. Understanding of endocarditis risk improves compliance with prophylaxis. Rev Port Cardiol. 2002; 21: 939-51.

2. Bozkurt AK, Oztunc F, Akman C., et al. Multiple pulmonary artery aneurysms due to infective endocarditis. Ann Thorac Surg. 2003; 75: 593-6.

3. Brooks N. Prophylactic antibiotic treatment to prevent infective endocarditis: new guidance from the National Institute for Health and Clinical Excellence. Heart 2009; 95: 774-780 (7)

4. Bulat DC, Kantoch MJ. How much do parents know about their children's heart condition and prophylaxis against endocarditis? Can J Cardiol 2003 Apr;19: 501- 6 .

5. Consensus on Timing of Intervention for Common Congenital Heart Diseases.
WORKING GROUP ON MANAGEMENT OF CONGENITAL HEART DISEASES IN INDIA. Indian Pediatrics. 2008; 45: 117-126

6. Hufnagel G., Pankuweit S., Richter A., et al. The European Study of Epidemiology and Treatment of Cardiac Inflammatory Diseases (ESETCID). First Epidemiological Results Die European Study of Epidemiology and Treatment of Cardiac Inflammatory Diseases (ESETCID) - Erste epidemiologische Ergebnisse. Herz. 2000; 25: 279-285.

7. Suryati BA, Watson M. Staphylococcus aureus bacteraemia in children: a 5-year retrospective review. J Paediatr Child Health. 2002; 38: 290-4.

8. Tokuda Y, Matsumoto M, Sugita T., et al. Methicillin-resistant Staphylococcus aureus endocarditis following repair of tetralogy of Fallot. Pediatr Cardiol 2002;23: 564-5.

9. Venkatesan C., Wainwright M. Pediatric Endocarditis and Stroke: A Single Center Retrospective Review of Seven Cases. Pediatr Neurol. 2008; 38: 243-247

10. Wilson W., Taubertat K., Gewitz M., al. Prevention of Infective Endocarditis. Guidelines From the American Heart Association: A Guideline From the American Heart Association Rheumatic Fever, Endocarditis, and Kawasaki Disease Committee, Council on Cardiovascular Disease in the Young, and the Council on Clinical Cardiology, Council on Cardiovascular Surgery and Anesthesia, and the Quality of Care and Outcomes Research Interdisciplinary Working Group. Circulation. 2007;116:1736-1754.

\section{Corresponding Author: \\ Lachezar Marinov MD,PhD \\ Department of Pediatrics \\ Medical University "Prof. Dr Paraskev Stoyanov" \\ 55 Marin Drinov Str., 9000 Varna, Bulgaria \\ Tel.: +359/899670 525; e-mail: marrinovs@abv.bg}

